\title{
2D Analytical Model of Armature Reaction Magnetic Field Distribution in Slotless Permanent-Magnet Linear Tubular Machines
}

\author{
A. Ghaffari \\ Department of Electrical and Electronics Engineering, Shiraz University of Technology, Shiraz, I.R. Iran \\ E-mail: a.ghaffari@sutech.ac.ir
}

Received: 4 April 2020; Accepted: 4 June 2020; Available online: 10 August 2020

\begin{abstract}
This paper presents a 2D analytical model for predicting the magnetic flux density distribution in slotless permanent-magnet (PM) linear tubular (PMLT) motors due to armature reaction effects based on the sub-domain method. According to this method, the machine cross-section is divided into the six sub-regions and Maxwell partial differential equations (PDEs) are formed in each sub-region. Solving these PDEs leads to defining the magnetic vector potential in each sub-region and applying curl on the calculated magnetic vector potential results in determining the magnetic flux density components. Eventually, the extracted results are compared with those of the finite-element method (FEM) to confirm the accuracy of the described analytical model. The results reveal that the presented analytical model is a suitable candidate for predicting the magnetic flux density components of the slotless PMLT motors in a shorter time.
\end{abstract}

Keywords: Analytical model; Armature reaction; Linear tubular motor; Maxwell equations; Sub-domain method.

\section{Introduction}

Various industrial and technological applications demand a linear motion that leads to developing linear machines in the past decades due to their unique advantages. The main advantage of these types of electrical machines is removing mechanical mechanisms. The mechanical mechanisms such as crankshafts and gears lead to increasing the losses and reducing the system agility and dynamic performance. Also, removing the mechanical mechanism results in having reliable and simple motion and the frictional wear is generated only in bearings [1][3]. According to the excitation, the linear machines are categorized into various groups and PM linear synchronous machines (PMLSMs) due to their considerable advantages such as high power density and low volume are extensively used in high speeds with high precision applications, transportation, factory automation systems and semiconductor manufacturing [4],[5].

The PMLSMs are mainly divided into the PM linear planar [6] and tubular [7] motors where in the tubular ones the winding are wound around the motor and the demerit of the end-turn effects are eliminated. Moreover, the radial force between the PMs and the stator cores in the PM linear tubular motor is nearly zero due to the symmetrical structure which is demonstrated in Fig.1. The stator structure in the linear PM motor is divided into the slotted and slotless structures where the detent force in the slotless stator structure is about zero. Also, the cost of winding in the slotless motor is lower than this cost in the slotted one [8].

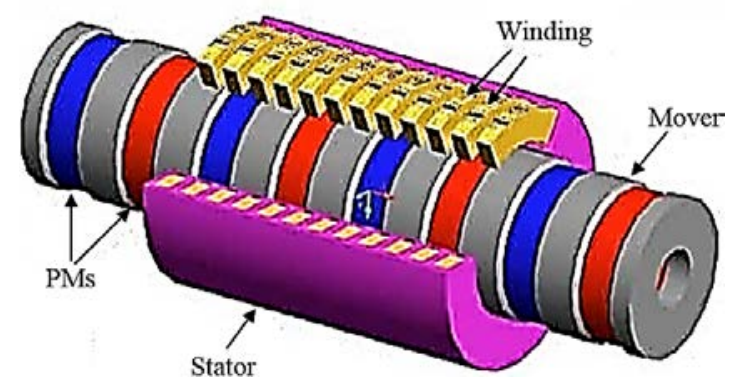

Fig. 1. The configuration of the PMLT motor

According to the eminent advantages of PM linear motors, it is necessary to determine an accurate model for predicting their behavior. So, the analytical and numerical models are developed to explain the behavior of the PM linear motors. The numeric models such as FEM, have high accuracy and these models are useful for considering geometric details and the nonlinearity of magnetic material [9]-[11]. But, the simulation time is the main 
disadvantage of the numerical models and these models are time-consuming in the design and optimization issues including too many iterations. Therefore, the analytical models, if possible, are preferred compare with the numerical ones due to their three privileges. The first benefit is related to the simulation time and this benefit is highlighted in the optimization step that is an essential issue for the design stage [12],[13]. Secondly, the analytical models provide a better sense of the system behavior by defining all the relevant equations and it is possible to realize the main parameters for controlling the output. The flexibility of the analytical model in terms of changing the design parameters is realized as the third advantage of these models where it is necessary to remodel the machine configuration to apply these changes in the numerical models[14]-[16]. Therefore, various researches have been concentrated on the analytical model in electrical machines, such as the PMLT motors under the study, since the past decades [17]-[21]. For instance, Wang J et al. [17] described the analysis, design, and experimental characterization of three-phase tubular modular permanent-magnet machines equipped with quasi-Halbach magnetized magnets where the effects of armature currents were not investigated in this paper. Wang J et al. [18] also prepared a new paper for analyzing the armature reaction field and defining the optimum design of the tubular PM machine. However, the permeability of cores in this paper was assumed infinite and there was no sense about the magnetic flux density distribution in cores. In [19] the analysis and experimental verification of a short-stroke, single-phase, quasi-Halbach magnetized tubular permanent magnet motor was described in which the magnets were mounted on a nonmagnetic support tube. Analytical solutions for the magnetic field distribution in the airspace and magnet regions were established in the cylindrical coordinate system and the flux density distribution in cores was not investigated in this paper [19]. The semi-analytical field calculation of the armature reaction in brushless tubular PM actuators with rectangular slots was reported in [20] where the permeability of cores is assumed infinite and the magnetic flux density components in cores were not studied. Meessen K. J et al. [22] explained the effects of changing the magnet shape of PMs in a slotless tubular actuator where the effects of armature currents were not determined and the flux density distributions in cores were not investigated due to assuming infinite permeability of cores. Therefore, an accurate model is required for calculating the flux density distribution in all regions of the PM tubular motors.

The main contribution of this paper is to explain an accurate analytical model of the presented PMLT motors for predicting the magnetic flux density components due to armature currents in all regions of the machine based on the sub-domain method.

\section{Proposed method}

\subsection{Assumptions}

The presented slotless PMLT motor is illustrated in Fig. 2 and all six sub-regions (i.e. exterior $(e)$ stator $(s)$, winding $(w)$, air-gap $(a)$, PMs and mover $(m)$ are shown in this figure. According to the machine structure, the polar coordinate is selected for modeling the motor under the study.

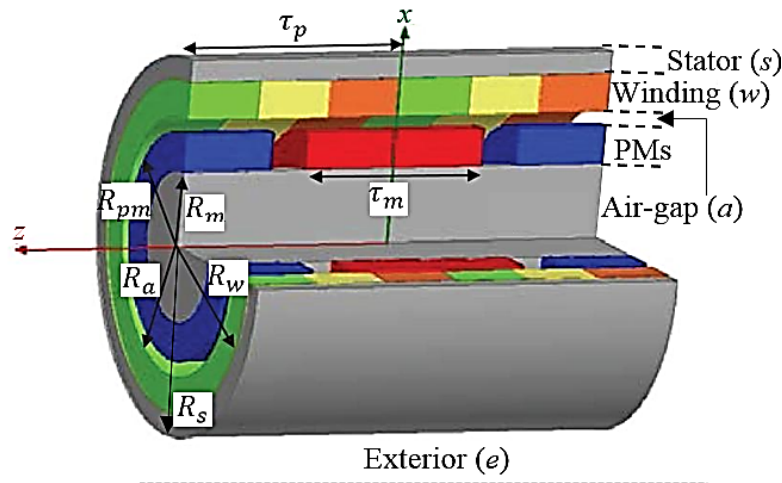

$\tau_{p}, \tau_{m}, R_{m}, R_{p m}, R_{a}, R_{w}$ and $R_{s}$ are pole pitch, PMs width, the radius of mover, PM, air-gap, winding and stator radius, respectively.

Fig. 2. The slotless PMLT motor under the study and the related sub-regions

Based on the sub-domain method, it is necessary to define the relevant assumptions and Maxwell PDEs in each sub-region are formed based on these assumptions. The pertinent assumptions for obtaining the analytical model of the studied motor are listed as follows:

a) The machine has infinite axial length.

b) The magnetic flux is originated due to only armature current.

c) The magnetic flux density vector has only radial and axial components. Also, the magnetic flux density vector and magnetic vector potential are independent of $\theta$. 
d) Magnetic vector potential has only $\theta$ component which is a function of radial (r) and axial position (z).

e) 6- The eddy current reaction is neglected.

f) 7- Current density vector, $\boldsymbol{J}$, has only $\theta$ component which is a function of $z$ and time (t).

\subsection{Maxwell PDEs in each sub-region}

According to the explained sub-regions, two main categories can be defined for the extracted Laplace and Poisson PDEs in the PMLT motor as follow:

$$
\nabla^{2} \boldsymbol{A}^{\boldsymbol{u}}=0 \quad u=m, P M, a, s, e
$$

$$
\nabla^{2} \boldsymbol{A}^{\boldsymbol{w}}=-\mu_{0} \boldsymbol{J}
$$

where $\mu_{0}$ is free space permeability. $\nabla^{2}$ is the laplacien operator and in the polar coordinate this operator is determined in the follow expression form:

$$
\nabla^{2} A=\left[\frac{1}{r} \frac{\partial}{\partial r}\left(r \frac{\partial}{\partial r}\right)+\frac{\partial^{2}}{\partial z}\right] A_{\theta}(r, z)
$$

To obtain the analytical model based on sub-domain method it is necessary to describe the armature reaction currents. In the q phases slotless PMLT machines the applied currents are defined as:

$$
i_{\gamma}(\mathrm{t})=\sum_{\xi} I_{\xi} \sin \left(\xi\left(p \frac{v}{L_{z}} \pi t-\frac{2 \pi(\gamma-1)}{q}\right)+\beta_{\xi}\right), \gamma=1,2, \ldots, q
$$

where $I_{\xi}, \beta_{\xi}, v, p$ and $L_{z}$ are the input peak current, phase shift of $\xi^{\text {th }}$ harmonic of the phase currents, the velocity of the mover, number of pole-pairs and stator length, respectively. According to the explained armature currents the current density Fourier series expansions is presented as:

$$
J_{\theta}(z, t)=\sum_{n=1}^{k} J_{1 n} \sin \left(\varpi_{n} z\right)+J_{2 n} \cos \left(\varpi_{n} z\right)
$$

where $\varpi_{n}=\frac{n \pi}{\tau_{p}}, n$ is maximum harmonic orders, $J_{1 n}$ and $J_{2 n}$ are current density Fourier series components that are described as:

$$
\begin{aligned}
& \left.J_{1 n}=-\frac{2 N_{t}}{\frac{\tau_{p}}{3}\left(R_{w}-R_{a}\right)} \frac{\cos \left(\frac{(\mathrm{q}+1) n \pi}{2 q}\right)-\cos \left(\frac{(\mathrm{q}-1) n \pi}{2 q}\right)}{n \pi} \times i_{\hbar}(t)+\sum_{\substack{\wp=1, \wp \neq \hbar}}^{(q-1) / 2} i_{\wp}(t) \cos \left(\frac{(q-r) n \pi}{q}\right)+\sum_{\substack{\mathfrak{J}=1, \mathfrak{s} \neq \hbar}}^{(q-1) / 2} i_{w}(t) \cos \left(\frac{(q+\Im) n \pi}{q}\right)\right] \\
& J_{2 n}=-\frac{2 N_{t}}{\frac{\tau_{p}}{3}\left(R_{w}-R_{a}\right)} \frac{\cos \left(\frac{(q+1) n \pi}{2 q}\right)-\cos \left(\frac{(q-1) n \pi}{2 q}\right)}{n \pi} \times \sum_{\varsigma=1}^{q} i_{\varsigma}(t) \sin \left(\frac{2(\varsigma-1) n \pi}{q}\right)
\end{aligned}
$$

where $N_{t}$ is the number of turns per coil, $\hbar$ is the phase by symmetrical distribution with respect to the $z$-axis. Fig. 3 presents the three phases winding currents and the applied current can be determined as:

$$
\begin{aligned}
& i_{a}(t)=I_{m} \sin \left(\frac{v}{\tau_{p}} \pi t\right) \\
& i_{b}(t)=I_{m} \sin \left(\frac{v}{\tau_{p}} \pi t-\frac{2 \pi}{3}\right) \\
& i_{c}(t)=I_{m} \sin \left(\frac{v}{\tau_{p}} \pi t+\frac{2 \pi}{3}\right)
\end{aligned}
$$


where $I_{m}$ is the peak of applied armature current.

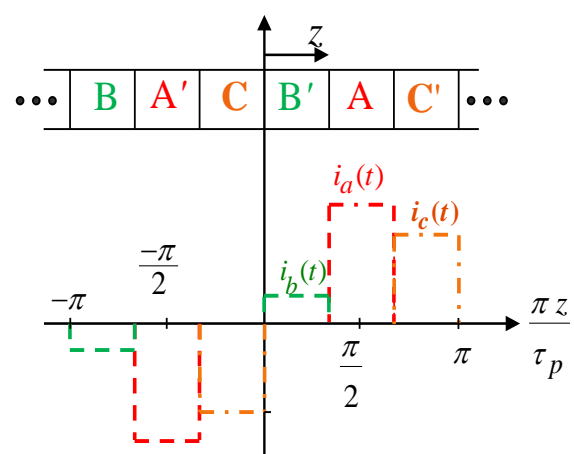

Fig. 3. Three phases winding of the presented slotless PMLT motor

Therefore, applying Eqs. (4)-(10), leads to obtaining the following Fourier series components for 3 phases machine:

$$
\begin{aligned}
& \left.J_{1 n}=-\frac{2 N_{t}}{\frac{\tau_{p}}{3}\left(R_{w}-R_{a}\right)} \frac{\cos \left(\frac{2 n \pi}{3}\right)-\cos \left(\frac{n \pi}{3}\right)}{n \pi} \times i_{a}(t)+i_{b}(t) \cos \left(\frac{2 n \pi}{3}\right)+i_{c}(t) \cos \left(\frac{2 n \pi}{3}\right)\right] \\
& J_{2 n}=-\frac{2 N_{t}}{\frac{\tau_{p}}{3}\left(R_{w}-R_{a}\right)} \frac{\cos \left(\frac{2 n \pi}{3}\right)-\cos \left(\frac{n \pi}{3}\right)}{n \pi} \times\left[-i_{b}(t) \sin \left(\frac{2 n \pi}{3}\right)+i_{C}(t) \sin \left(\frac{2 n \pi}{3}\right)\right]
\end{aligned}
$$

According to the extracted PDEs in each sub-region and the current density Fourier series expansion, the magnetic vector potential in each sub-region is defined as follow:

$$
\begin{gathered}
A_{\theta}^{u}(r, z)=\sum_{n=1}^{k} \frac{1}{\varpi_{n}}\left[\left(a_{n}^{u} I_{1}\left(\varpi_{n} r\right)+b_{n}^{u} K_{1}\left(\varpi_{n} r\right)\right) \cos \left(\varpi_{n} z\right)+\left(c_{n}^{u} I_{1}\left(\varpi_{n} r\right)+d_{n}^{u} K_{1}\left(\varpi_{n} r\right)\right) \sin \left(\varpi_{n} z\right)\right] \\
A_{\theta}^{w}(r, z)=\sum_{n=1}^{k} \frac{1}{\varpi_{n}}\left[\left(a_{n}^{w} I_{1}\left(\varpi_{n} r\right)+b_{n}^{w} K_{1}\left(\varpi_{n} r\right)\right) \cos \left(\varpi_{n} z\right)+\left(c_{n}^{w} I_{1}\left(\varpi_{n} r\right)+d_{n}^{w} K_{1}\left(\varpi_{n} r\right)\right) \sin \left(\varpi_{n} z\right)\right. \\
\left.+\frac{\mu_{0} \jmath_{2 n}}{\varpi_{n}} \cos \left(\varpi_{n} z\right)+\frac{\mu_{0} J_{1 n}}{\varpi_{n}} \sin \left(\varpi_{n} z\right)\right]
\end{gathered}
$$

where $I_{1}$ and $K_{1}$ are type one of the first and second kinds of modified Bessel function. Applying curl on the obtained magnetic vector potential results in calculating the magnetic flux density components in each sub-region. Therefore, the normal $\left(B_{r}\right)$ and tangential $\left(B_{z}\right)$ components of the magnetic flux density are determined in the following expressions:

$$
\begin{aligned}
& B_{r}=-\frac{\partial A_{\theta}(r, z)}{\partial z} \\
& B_{z}=\frac{1}{r} \frac{\partial}{\partial r}\left(r A_{\theta}(r, z)\right)
\end{aligned}
$$

Noted that $I_{1}^{\prime}(x)=I_{0}(x)-\frac{1}{x} I_{1}(x), K_{1}^{\prime}(x)=-K_{0}(x)-\frac{1}{x} K_{1}(x)$ where $I_{0}$ and $K_{0}$ are the type zero of the first and second kinds of modified Bessel function, respectively.

\subsection{Boundary conditions}


The obtained magnetic flux density components include 20 unknown variables such as $\left\{b_{n}^{e}, d_{n}^{e}, a_{n}^{s}, b_{n}^{s}, c_{n}^{s}, d_{n}^{s}\right.$, $\left.a_{n}^{w}, b_{n}^{w}, c_{n}^{w}, d_{n}^{w}, a_{n}^{a}, b_{n}^{a}, c_{n}^{a}, d_{n}^{a}, a_{n}^{p m}, b_{n}^{p m}, c_{n}^{p m}, d_{n}^{p m}, a_{n}^{m}, c_{n}^{m}\right\}$. According to the geometry considerations and the defined coordinate system, some of these variables must be zero, (i.e. $a_{n}^{e}=c_{n}^{e}=b_{n}^{m}=d_{n}^{m}=0$ ). Therefore, it is necessary to explain 20 equations to determine the exact magnetic flux density expressions. Hence, the boundary conditions are utilized to predict the magnetic flux density distributions. Based on the magnetic boundary conditions, the normal component of the magnetic flux density is continuous at the interfaces between two adjacent sub-regions. Also, In the case of the source-free interface, the tangential component of the magnetic field intensity is continuous at that interface. So, the boundary conditions in the presented PMTL motor is defined as follow:

$$
\begin{aligned}
& \left.B_{r}^{i}(r, z)\right|_{r=R}=\left.B_{r}^{i+}(r, z)\right|_{r=R} \\
& \left.H_{z}^{i}(r, z)\right|_{r=R}=\left.H_{z}^{i+}(r, z)\right|_{r=R}
\end{aligned} \quad(i, i+, R)=\left\{\begin{array}{l}
\left(e, s, R_{s}\right),\left(s, w, R_{w}\right),\left(w, a, R_{a}\right), \\
\left(a, P M, R_{P M}\right),\left(P M, m, R_{m}\right)
\end{array}\right\}
$$

\section{Results and discussions}

In order to validate the described analytical model, the magnetic flux density components due to only armature currents are calculated for the case study. The obtained analytical results are compared with those of FEM to determine the accuracy of the extracted results. Table 1 lists the main design parameters of the PMLT motor under the study to predict the magnetic flux density in each sub-region. The analytical and numerical results are shown in Fig. 4. As it is evident, the numerical results confirm the accuracy of the derived analytical model. Therefore, the numerical model can be replaced by the defined analytical model for predicting the magnetic flux density distribution in all media of the presented PMLT motor to save time. Moreover, the simulation time reveals that the analytical model is 14 times faster than the numerical one and this benefit is highlighted in the design and optimization issues including several thousands of optimization iterations.

Noted that, the magnetic flux density amplitude in each sub-region is not considerable due to the large magnetic air-gap height in the slotless stator structure of the presented linear motor.

Table 1. The main design parameters of the studied PMTL motor

\begin{tabular}{cc}
\hline Parameters & Value \\
\hline Number of pole-pairs & 2 \\
Number of winding phase & 3 \\
PM Remanence flux density (T) & 0 \\
Motor axial length (mm) & 240 \\
PM width (mm) & 40 \\
Mover radius (mm) & 10 \\
PM radius (mm) & 20 \\
Air-gap radius (mm) & 22 \\
Winding radius (mm) & 35 \\
Stator radius (mm) & 50 \\
Motor speed (m/s) & 1 \\
PM permeability & 1.08 \\
Number of turns per coil & 150 \\
Peak of armature current (A) & 5 \\
\hline
\end{tabular}

\section{Conclusions}

In this paper, a 2D analytical model has been developed for determining an accurate model of slotless PM linear tubular motor. The defined analytical model was employed to predict the effects of armature currents on magnetic flux density distribution in all sub-regions of the motor based on the sub-domain method. Moreover, finite permeability of all sub-regions and its corresponding impacts have been explained to calculate the magnetic flux density in cores. The normal and tangential components of the magnetic flux density were analytically extracted based on solving the Maxwell equations. The FEM was employed to confirm the accuracy of the presented model. Also, the simulation time explained the most highlighted benefit of the utilized analytical model where this model was 14 times faster than the numerical one in the case study. Therefore, the obtained model can be realized as a proper candidate in the design and analysis of the slotless PMLT motor. Based on the slotless structure of the motor under the study, the magnetic flux density components were not notable but these small amounts are necessary to provide the output power. 


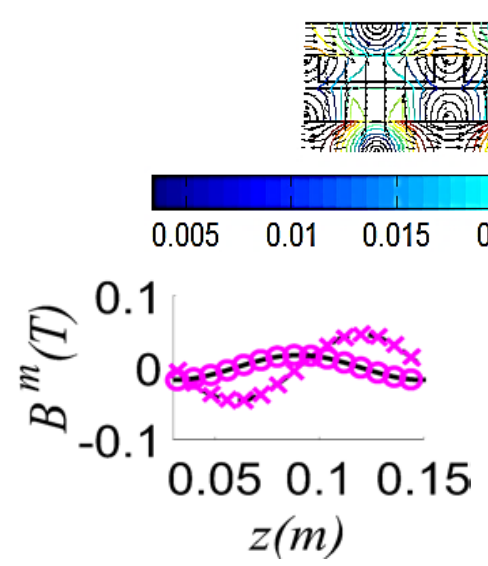

(a): Mover

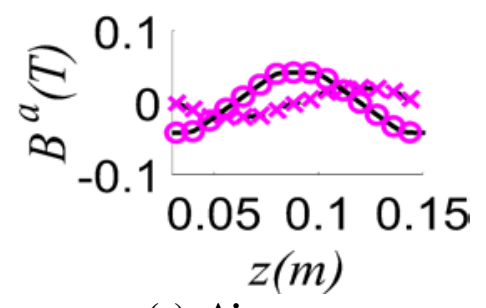

(c): Air-gap

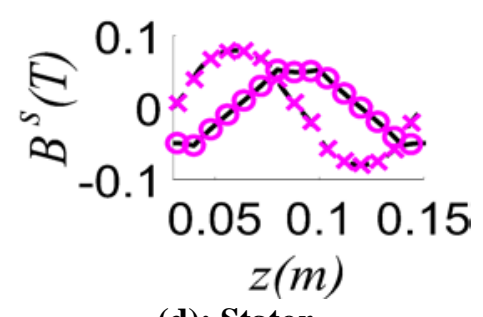

(d): Stator

- - Analytical results of the axial component of the flux density

- Analytical results of the radial component of the flux density

O Numerical results of the radial component of the flux density

$\times$ Numerical results of the axial component of the flux density

Fig. 4. Magnetic flux density components in all sub-regions of the motor under the study due to only armature reaction

\section{References}

[1] Gieras JF, Piech ZJ. Linear synchronous motors. transportation and automation systems. CRC Press LLC. 2000.

[2] Ghaffari A, Khalili F, Vahaj A.A, Ghaffari H, Mahmoudi A. 2-D analytical model for slotless double-sided outer armature permanent-magnet linear motor. Progress in Electromagnetics Research C. 2020;101:173-186.

[3] Ghaffari A, Rahideh A, Ghaffari H, Vahaj A.A, Mahmoudi A. Comparison between 2-D and 0-D analytical models for slotless double-sided inner armature linear permanent magnet synchronous machines. International Transaction on Electrical Energy Systems. 2020;e12509.

[4] Wang H, Li J, Qu R, Lai J, Huang H, Liu H. Study on high efficiency permanent magnet linear synchronous motor for maglev. IEEE Transactions on Applied Superconductivity. 2018;28(3):1-5.

[5] Wen C, Liu J, Wang W, Liu J, Zhao Z, Liu J. Research on improved permanent magnet linear synchronous motor for direct-drive application. IEEE Transactions on Magnetics. 2019;55(10):1-7.

[6] Boduroglu A, Gulec M, Demir Y, Yolacan E, Aydin M. A new asymmetric planar V-shaped magnet arrangement for a linear pm synchronous motor. IEEE Transactions on Magnetics. 2019;55(7):1-5.

[7] Huang XZ, Li J, Tan Q, Qian ZY, Zhang C, Li L. Sectional combinations of the modular tubular permanent magnet linear motor and the optimization design. IEEE Transactions on Industrial Electronics. 2018;65(12):9658-9667.

[8] Min SG, Sarlioglu B. Analytical calculation of back EMF waveform for linear PM motors in slotted and slotless structures. IEEE Transactions on Magnetics. 2017;53(12):1-10.

[9] Vese I, Marignetti F, Radulescu MM. Multiphysics approach to numerical modeling of a permanent-magnet tubular linear motor. IEEE Transactions on Industrial Electronics. 2010;57(1):320-326.

[10] Ghaffari A. Analytical model of the slotless double-sided axial flux permanent-magnet brushless machines. Journal of Modeling and Optimization. 2020;12(1):38-50.

[11] Xu L, Lin M, Fu X, Li N. Analysis of a double stator linear rotary permanent magnet motor with orthogonally arrayed permanent magnets. IEEE Transactions on Magnetics. 2016;52(7):1-4. 
[12] Ghaffari A, Rahideh A, Moayed-Jahromi H, Vahaj AA, Mahmoudi A, Soong WL. 2-D analytical model for outer-rotor consequent-pole brushless PM machines. IEEE Transactions on Energy Conversion. 2019;34(4):226-2234.

[13] Rahideh A, Ghaffari A, Barzegar A, Mahmoudi A. Analytical model of slotless brushless PM linear motors considering different magnetization patterns. IEEE Transactions on Energy Conversion. 2018;33(4):17971804.

[14] Ghaffari A. 2-D analytical model for predicting magnetic flux distribution in slotless single-sided axial flux permanent-magnet synchronous machines. Journal of Modeling and Optimization. 2019;11(2):97-105

[15] Vahaj AA, Rahideh A, Moayed-Jahromi H, Ghaffari A. Exact two-dimensional analytical calculations for magnetic field, electromagnetic torque, UMF, Back-EMF, and inductance of outer rotor surface inset permanent magnet machines. Mathematical and Computational Applications. 2019;24(1):24.

[16] Min SG, Sarlioglu B. Analytical prediction and multiconstrained nonlinear optimization of slotted linear PM motors taking into account two-dimensional end effects. IEEE Transactions on Industrial Electronics. 2020;67(4):2965-2976.

[17] Wang J, Howe D. Tubular modular permanent-magnet machines equipped with quasi-Halbach magnetized magnets-part I: magnetic field distribution, EMF, and thrust force. IEEE Transactions on Magnetics. 2005;41(9):2470-2478.

[18] Wang J, Howe D. Tubular modular permanent-magnet machines equipped with quasi-Halbach magnetized magnets-part II: armature reaction and design optimization. IEEE Transactions on Magnetics. 2005;41(9):2479-2489.

[19] Ibrahim T, Wang J, Howe D. Analysis and experimental verification of a single-phase, quasi-halbach magnetized tubular permanent magnet motor with non-ferromagnetic support tube. IEEE Transactions on Magnetics. 2008;44(11):4361-4364.

[20] Gysen BLJ, Meessen KJ, Paulides JJH, Lomonova EA. Semi-analytical calculation of the armature reaction in slotted tubular permanent magnet actuators. IEEE Transactions on Magnetics. 2008;44(11):3216-3216.

[21] Huang XZ, Li J, Tan Q, Qian ZY, Zhang C, Li L. Sectional combinations of the modular tubular permanent magnet linear motor and the optimization design. IEEE Transactions on Industrial Electronics. 2018;65(12):9658-9667.

[22] Meessen KJ, Gysen BLJ, Paulides JJH, Lomonova EA. Halbach permanent magnet shape selection for slotless tubular actuators. IEEE Transactions on Magnetics. 2008;44(11):4305-4308.

(c) 2020 by the author(s). This work is licensed under a Creative Commons Attribution 4.0 International License (http://creativecommons.org/licenses/by/4.0/). Authors retain copyright of their work, with first publication rights granted to Tech Reviews Ltd. 